nucleus periphery. Intranuclear bodies of $5 \mu \mathrm{m}$ in size contain RNA (RiboGreen), coilin (R288) and FLASH. They are attached to chromosomes. The presence of coilin suggests these structures are some kind of Cajal bodies. However FLASH protein is a marker of histone locus bodies. At the same time zebra finch oocyte nucleus do not contain 'protein bodies' which are common for chaffinch. The lampbrush chromosomes lie in the center of nucleus and do not contact inner nuclear membrane. The most peculiar one is germline-restricted chromosome (GRC) which is the biggest chromosome in karyotype. It is actively transcribed but presents only in gametes. Lampbrush form of this chromosome have obvious condensed 'belts'. There are small coilin positive bodies attached to this 'belts'. We see, that even members of the same order but different families have various intranuclear structures.

Research Resource Centre "Chromas" of Saint Petersburg State University.

doi: http://dx.doi.org/10.7124/bc.000A03

\section{W-1. ROS-induced PML nuclear bodies biogenesis}

Chengchen WU, Pierre Bercier, Shirine Benhenda, Valérie Lallemand-Breitenbach, Hugues de The

College de France U1050, INSERM, CNRS

chengchen.wu@college-de-france.fr

Promyelocytic leukemia nuclear bodies (PML-NBs) are stress-sensitive membrane-less organelles organized by PML, a tumor suppressor protein. PML-NBs are involved in a wide array of biological processes, such as apoptosis, senescence, DNA repair, epigenetic control, control of oncogenesis. The two-step PML-NBs assembly consists first in PML scaffold polymerization in shell-like bodies, then interaction with huge diversity of unrelated proteins recruited in the inner-core. PML-NBs are considered as privileged sites for post-translational modifications (PTM), especially sumoylation. The oxidative stress like that induced by arsenic promotes PML intermolecular disulfide bonds formation and sumoylation, but the mechanism of PML-NBs assembly and dynamics upon stress or at basal level is still poorly understood. Our aim is to unravel the mechanism of PMLNBs biogenesis, assembly and function through the interdisciplinary approach combing molecular and cell biology perspective, and biophysical insight of PML dynamics in living cells to shed light on the way in which PML biological activity modulates the NBs organization. In living cells, we observed PML NBs fusion and fission events. Quantitative analyses of PML dynamics show that PML behaves as a viscous fluid. As2O3 treatment dramatically decreases the diffused PML in nucleus with time, and the dynamic turnover of PML at NBs (FRAP) is abolished. These data revealed that PML has properties at the frontier of liquid and gel, which is strongly modulated by oxidative stress. Upon oxidative stress, PML is covalently bound through intermolecular disulfide bonds, which are formed by the oxidation of cysteines in PML. We show that cysteine mutants in the conserved RBCC domain of PML B2 box are not able to form normal PML-NBs and further sumoylation. These mutants have more diffused PML distributed in the nucleus, but no difference can be observed after $\mathrm{As} 2 \mathrm{O} 3$ 
treatment, suggesting oxidative stress-induced di-sulfide bonds are also impaired in these mutants. We conclude that the oxidation of B2 box in PML plays a key role in PML-NBs assembly and sumoylation. We also identified a specific C-terminal cysteine in PML that participates in the formation of intermolecular disulfide bonds, yet not essential for PML-NBs assembly. This oxidized PML might contribute to maintain a specific redox environment in cells.

doi: http://dx.doi.org/10.7124/bc.000A04

\section{Z-1. Lysine methyltransferase SETDB1 and regulation of the three-dimensional organization of the genome during lung cancer progression}

V. Zakharova, G. Tellier, L. Del Maestro, V. Joliot and S. Ait-Si-Ali.

CNRS UMR7216 "Epigénétique et Destin Cellulaire" Université Paris Diderot Paris 7

zakharova.vlada@gmail.com

SETDB1 histone H3 lysine 9 (H3K9) methyltransferase (KMT) is known to be involved in tumorigenesis; however, the exact mechanisms of this involvement remain mainly unknown. SETDB1 regulates chromosomal conformation (Jiang et al., 2017); we recently found that SETDB1 interacts and could methylate members of the Cohesin complex. CTCF and Cohesin complex delimit Topologically Associated Domains (TADs) through direct binding at TAD boundaries. CTCF binding to chromatin is inhibited by $\mathrm{H} 3 \mathrm{~K} 9$ - and DNAmethylation. H3K9 trimethylation (H3K9me3) is a marker of metastasis in lung cancer patients, where SETDB1 gene is amplified. SETDB1 overexpression was associated with elevated cell growth and invasiveness of lung cancer cells (Rodriguez-Paredes et al., 2014). On the other hand, SETDB1 could act as a metastasis suppressor strongly downregulated in highly metastatic lung cancer cells. Aim: Here, we address the role of SETDB1 in the regulation of $3 \mathrm{D}$ genome architecture and gene expression patterns in an epithelial lung cancer cell line with different expression levels of SETDB1 (normal, low or high). Results: We found that SETDB1 regulates the epithelialmesenchymal transition that is a crucial in cancer progression and metastasis. We plan to perform CTCF, SETDB1 and H3K9me3 ChIPSeq assays combined with Bis-Seq to study DNA methylation at the CTCF binding sites in our cellular models. We will next correlate Hi-C maps with the defined epigenetic landscape. Conclusions: We expect that SETDB1 interacts with the Cohesin complex and affects CTCF occupancy at TAD boundaries, impacting 3D genome architecture and gene expression during during cancer progression.

doi: http://dx.doi.org/10.7124/bc.000A05

\section{Z-2. Investigating the nucleolar epigenetic code at ultrastructural level}

$\underline{\text { L. Zannino }}^{1}$, V. Bertone ${ }^{2}$, S. Siciliani ${ }^{1}$,

L. Saia ${ }^{1}$, M. Biggiogera ${ }^{1}$

${ }^{1}$ Laboratory of Cell Biology and Neurobiology, Department of Biology and Biotechnology, University of Pavia, Italy; ${ }^{2}$ Laboratory of Comparative 